\title{
Application of an Efficient Method for Isolation of Mitochondria from Biological Samples
}

\author{
Jin Hyun Jun ${ }^{1,2}$, Jihyun Kim ${ }^{1,2}$, Kyung Tae Kim ${ }^{3}$ and Ho Joong Sung ${ }^{1,2, \uparrow}$ \\ ${ }^{l}$ Department of Biomedical Laboratory Science, College of Health Science, Eulji University, \\ Seongnam 461-713, Korea \\ ${ }^{2}$ Department of Senior Healthcare, BK21 Plus Program, Graduated School, Eulji University, \\ Daejeon 301-746, Korea \\ ${ }^{3}$ Alphagene Co., Ltd., Seongnam 461-713, Korea
}

\begin{abstract}
Mitochondria play a crucial role in many essential biological events by way of the electron transport chains and intermembrane proteins that they contain. Abnormalities in the mitochondria are strongly correlated with the development of diseases such as atherosclerosis, cancer, and diabetes. However, the study of mitochondria has been referred to as 'labor-intensive' because of the difficulty in isolating the organelles from their various sources, which can include cultured cells and tissues. Multiple companies provide mitochondria isolation kits, and it is possible for investigators to use different kits and apply different protocols depending on the source of the mitochondria. Therefore, we focused on producing an isolation buffer that could be applied to both cultured cells and tissues, and optimized an isolation protocol that could be used with both. Specifically, we adjusted the buffer condition that can be applied to human cervical cancer cells, fibroblasts, and tissues such as mouse liver and spleen. We also optimized the protocol to improve the efficacy and efficiency of the steps involved in the isolation of mitochondria. These methodological improvements may contribute to advanced research by allowing investigators to overcome the difficulties involved in isolation of mitochondria from biological samples.
\end{abstract}

Key Words: Mitochondria, Isolation, Cells, Tissues, Optimized protocol

Mitochondria are key organelles that regulate many different signaling pathways, including those involved in the life and death of cells (Frezza et al., 2007). In the mitochondria of aerobic organisms, electron transport chains (ETCs) serve as a site for production of cellular energy by oxidative phosphorylation, a process in which molecular oxygen is used to generate adenosine triphosphate (ATP). During oxidative phosphorylation, reactive oxygen species (ROS) are generated in Complexes I and III of the ETC by

\footnotetext{
* Received: August 4, 2014 / Revised: September 11, 2014

Accepted: September 11, 2014

${ }^{\dagger}$ Corresponding author: Ho Joong Sung. Department of Biomedical Laboratory Science, College of Health Science, Eulji University 553 Sanseong, Soojeong, Seongnam, Gyeonggyi 461-713, Korea. Tel: +82-31-740-7306, Fax: +82-31-740-7425 e-mail: hjsung@eulji.ac.kr

(C)The Korean Society for Biomedical Laboratory Sciences. All rights reserved.
}

mitochondrial biogenesis. Overproduction of ROS is considered a major cause of several diseases, including atherosclerosis, cancer, and diabetes (Waris and Ahsan, 2006). Mitochondria also regulate apoptosis, releasing 'death signals' that control the mortality of cells. Specifically, cytochrome $c$ and other intermembrane space proteins are released and these activate effector caspases that are essential for apoptosis. To regulate apoptosis, mitochondria interact with another organelle, the endoplasmic reticulum (ER). They also interact with the nucleus to modify gene expression according to metabolic demand and cellular stress levels (Runkel et al., 2014).

Properly functioning mitochondria are important to many organisms, and there is growing evidence that mitochondrial dysfunction increases the incidence of diseases such as 
cancer and heart failure. For example, mitochondrial dysfunction accelerates damage to DNA, which promotes genetic instability and drives tumorigenesis, and this can stimulate the development of cancer (Sung et al., 2010). The heart, composed of approximately $25 \%$ myocardial cells, contains mitochondria that regulate cardiac bioenergetics. The risk for cardiac disease is significantly increased in instances of respiratory chain dysfunctions (Schwarz et al., 2014). In particular, mitochondrial malfunction can cause cardiomyopathy, heart failure, and ischemia reperfusioninduced injuries.

As organisms age, the number of mitochondria as well as their functionality decreases. Thus, copy number of mitochondrial DNA (mtDNA) decreases, and the capacity of ETC reduces. Consequently, mutations in the mtDNA accumulate and production of ROS increases. The increase in mtDNA mutations and ROS production is thought to cause age-related diseases (Bratic and Larsson, 2014).

The study of mitochondria has become essential to determine the molecular mechanism of mitochondria-related diseases and to understand their critical role in energy metabolism. In order to investigate mitochondria, they must first be isolated in the intact form from target cells and tissues. Diverse methods are available for isolating mitochondria, and the method used depends on the type of cells and tissues used (Frezza et al., 2007). For example, based on the source of mitochondria, step-by-step procedures for isolation, including factors such as centrifugal force, time, and number, are often adjusted. In addition, it may be necessary to consider the composition of an isolation buffer depending on the origin of mitochondria. For example, the concentration of monosaccharides such as sucrose and mannitol in a buffer may differ with the origin of mitochondria.

To overcome the difficulties in mitochondria isolation, isolation kits have been developed and commercialized by multiple companies. Research into the mitochondria has been accelerated because of these isolation kits, but investigators often need to obtain different types of kit from the same company, and have to apply different protocols, depending on the source of the mitochondria.

Therefore, we focused on producing an isolation buffer
Table 1. Composition of the isolation buffer ( $\mathrm{pH} 7.4)$

\begin{tabular}{lc}
\hline \hline Reagents & Concentration $(\mathrm{mM})$ \\
\hline Sucrose & 250.0 \\
HEPES $^{*}$ & 10.0 \\
EGTA $^{* *}$ & 1.0 \\
PMSF $^{* * *}$ & 0.5 \\
\hline
\end{tabular}

*N-2-hydroxyethylpiperazine-N-2-ethane sulfonic acid,

**Ethylene glycol tetraacetic acid,

***Phenylmethanesulfonyl fluoride

The buffer was prepared on ice and stored at $-20^{\circ} \mathrm{C}$.

that could be applied to cultured cells and tissues, and followed this up by optimizing a protocol that could be used with both. To test our protocol, we purchased a commercially available mitochondria isolation kit and compared it with our method for efficiency. To protect the anonymity of the company and commercial kit, we do not provide the name of either.

First, we adjusted the isolation buffer conditions as shown in Table 1. All materials used to prepare the isolation buffer were purchased from Sigma-Aldrich (St. Louis, MO, USA). We were unable to verify the composition of the buffers supplied in the commercial kit and compare the contents with our buffer, because the company withheld this information. However, the isolation kit contained multiple buffers for use in isolating mitochondria from cultured cells. In addition, the manufacturer's protocol suggested the use of multiple buffers in a given order during the isolation procedure. Next, we optimized a protocol that could be applied to both cultured cells and tissues (Fig. 1). The aim of introducing this protocol was to isolate mitochondria by using a single buffer within 40 50 min. Additionally, all steps of the isolation protocol, except homogenization, would be identical regardless of the cultured cells and tissues used. To test the buffers and protocols, we isolated mitochondria from human cancer cells and fibroblasts. The human cervical cancer cell line HeLa was obtained from American Type Culture Collection (ATCC, Manassas, VA, USA). Human fibroblasts were kindly provided from Prof. Haekwon Kim (Seoul Women's University, Seoul, Korea). HeLa cells and fibroblasts were maintained and cultured in a tissue culture incubator at $37^{\circ} \mathrm{C}$ by using complemented 


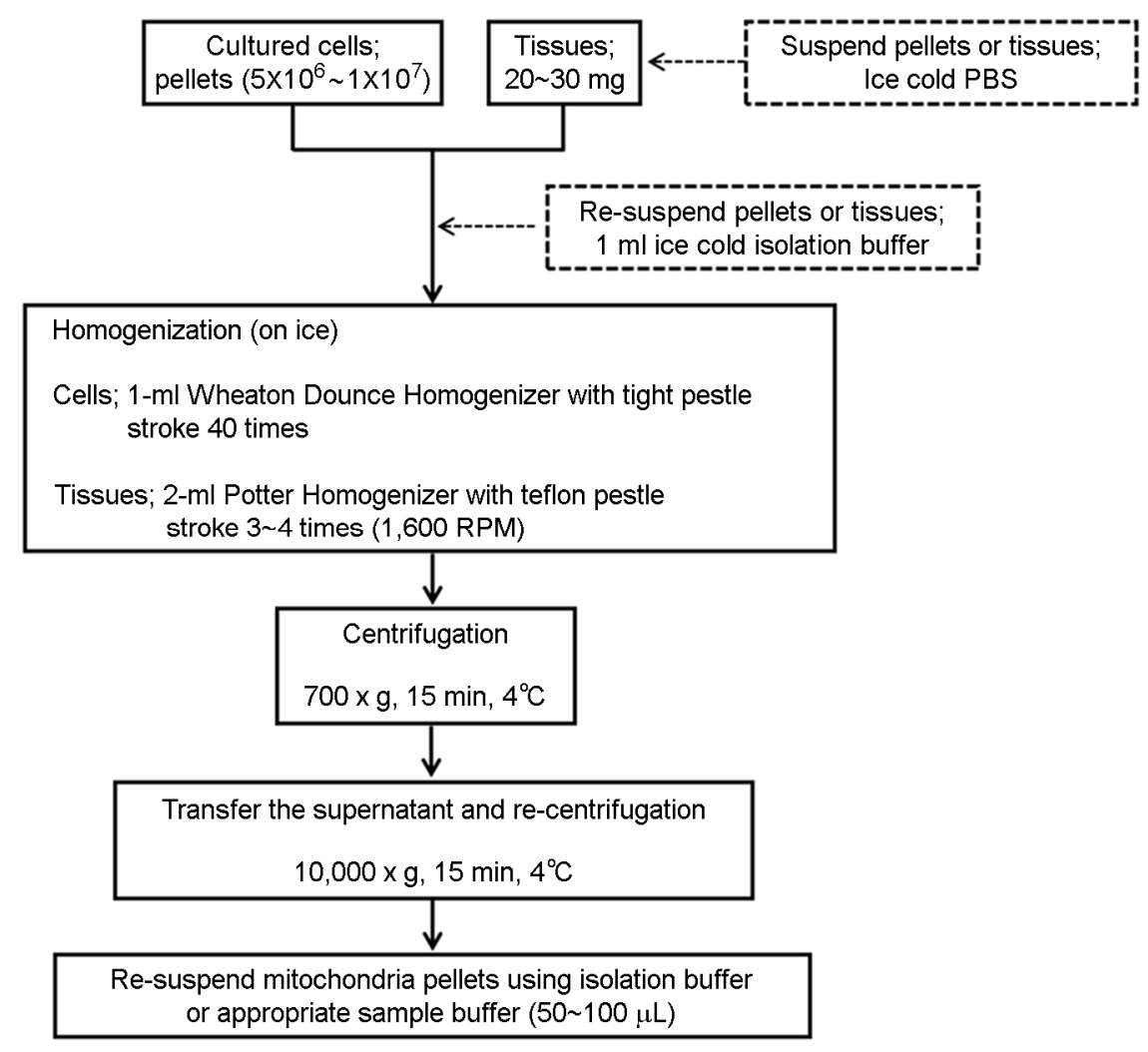

Fig. 1. Workflow for isolating mitochondria from cultured cells and tissues using an optimized isolation buffer. Cells in culture flasks were scraped with a rubber spatula, inoculated into $3 \sim 5 \mathrm{~mL}$ of culture medium, and pooled into a $15-\mathrm{mL}$ conical tube. After centrifugation at $300 \mathrm{~g}$ for $10 \mathrm{~min}$, cell pellets were suspended in ice-cold PBS. Isolation of mitochondria from cells was conducted according to the workflow. With respect to extraction of mouse tissue, a mouse was dissected immediately before mitochondria isolation, in accordance with the Institute's animal care guidelines. Following dissection, tissues were rinsed with ice-cold PBS to remove blood and the wet weight of the tissues was measured. We then isolated mitochondria from 20 to $30 \mathrm{mg}$ of tissue by using the procedure usually used for isolation from cultured cells. Note that all steps of isolation, except centrifugation, were performed on ice or at a temperature below $4^{\circ} \mathrm{C}$.

Dulbecco's modified Eagle's medium (DMEM) with 10\% fetal bovine serum (Life Technologies, Grand Island, NY, USA). Cells were maintained at up to $75 \%$ confluency to prevent stressed culture conditions. We isolated mitochondria from both cell lines by using our protocol and measured the amount of mitochondrial protein in protein sample buffers by using Bradford protein assay kit (Bio-Rad, Hercules, CA, USA). To determine whether the mitochondria were successfully isolated, we examined protein expression in a voltage-dependent anion channel (VDAC), which has been known to present in the mitochondrial outer membrane, by using the western blotting method. For the western blot, we used mouse monoclonal-VDAC antibody and goat antimouse antibody conjugated with horseradish peroxidase (HRP) (Mitosciences, Eugene, OR, USA) according to the manufacturer's protocol. We observed VDAC expression to follow a dose-dependent pattern in both HeLa cells and fibroblasts following mitochondria isolation, as shown in Fig. 2A. We performed mitochondria isolation simultaneously, by using our method as well as the commercial kit. We ensured that all experimental conditions such as confluency of cultured cells were maintained identical. Using the same amount of protein $(25 \mu \mathrm{g})$ from isolated mitochondria, we compared VDAC expression by western blotting, as shown in Fig. 2B. We found that VDAC expression was higher with our optimized method than with the suggested method from the kit. Also, in case of mitochondria isolation from fibroblasts, VDAC expression was significantly higher with our method. To test the ability of our method for isolating mitochondria from tissues 
A

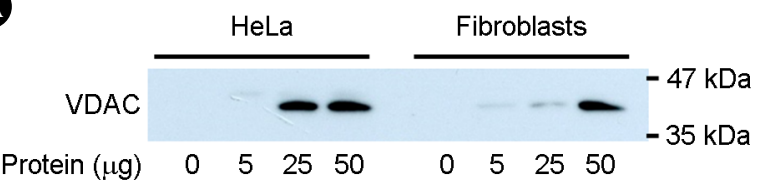

B

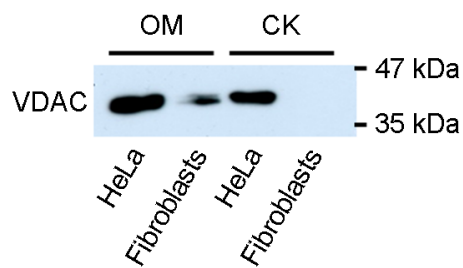

Fig. 2. Isolation of mitochondria from cultured cells by using an adjusted isolation buffer and an optimized protocol. (A) About $1 \times 10^{7}$ human cervical cancer cells (HeLa) and $5 \times 10^{6}$ human fibroblasts were prepared for mitochondria isolation. After isolation, the mitochondria were lysed in radioimmunoprecipitation assay (RIPA) buffer and the amount of total mitochondrial protein was measured by Bradford assay. Same amounts of protein from both HeLa cells and fibroblasts were applied in a dose-dependent manner, as shown in the figure. After SDS-PAGE, the proteins were transferred to nitrocellulose membranes. Membranes were incubated with $5 \%$ skimmed milk for $1 \mathrm{~h}$ at room temperature, followed by overnight incubation with anti-VDAC antibodies $(1: 3,000)$ at $4{ }^{\circ} \mathrm{C}$. The expression of VDAC (expected molecular weight, $39 \mathrm{kDa}$ ) in both cell lines was observed. Experiments were conducted at least three times, independently of each other. (B) Mitochondrial proteins from both cell lines were simultaneously prepared using our method (OM) and a commercial kit (CK). Thirty micrograms of protein from each cell line was used for western blotting. In the method provided for $\mathrm{CK}$, three different confidential buffers were used in sequential order. In addition, different centrifugal conditions were sequentially applied, as follows: $700 \mathrm{~g}\left(10 \mathrm{~min}, 4^{\circ} \mathrm{C}\right), 12,000 \mathrm{~g}(15$ $\left.\min , 4^{\circ} \mathrm{C}\right)$, and $12,000 \mathrm{~g}\left(5 \mathrm{~min}, 4^{\circ} \mathrm{C}\right)$. Experiments were conducted at least three times, independently of each other.

rather than cultured cells, we isolated mitochondria from the liver and spleen of a mouse. Again, we measured VDAC expression to determine the success of mitochondria isolation (Fig. 3). We found expression of VDAC in mouse tissues, and protein expression levels were higher when using our isolation method compared the method in the manufacturer's kit. Unexpectedly, we found that mitochondria could be isolated from mouse tissue by using a kit designed for use with 'cultured cells only'. The trade secrets of the company prevent us from comparing the ingredients of mitochondria isolation kits designed for use with cultured cells and tissues. Therefore, we could not compare the efficiency of our method, a for-cell-only kit, and a for-tissue-

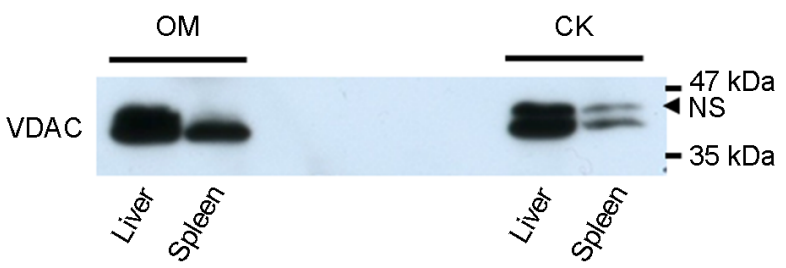

Fig. 3. Isolation of mitochondria from tissues by using an adjusted isolation buffer and an optimized protocol. Mouse liver and spleen tissues were prepared and $20 \mathrm{mg}$ of each tissue was used for isolation of mitochondria. Using our method $(\mathrm{OM})$ and a commercial kit (CK), isolation of mitochondria was conducted simultaneously. Twenty micrograms of mitochondrial proteins was used for western blotting. Experiments were performed multiple times. NS, applied to a representative image, denotes 'non-specific'.

only kit with respect to mitochondria isolation. However, we could argue, based on our results, that our optimized method might be appropriate to isolate mitochondria from mouse tissue.

In general, researchers have examined several aspects of the mitochondria, including structure, protein expression, and activity. We focused on protein expression in isolated mitochondria, and introduced a new optimized method that, our results suggest, could positively contribute to the study of mitochondria. Using our method, researchers could reduce the level of labor currently required to study mitochondria. To extend our investigation, we would examine the activity of mitochondria following isolation using our method and compare the results to those obtained using a commercial kit. This further work could help determine if our method is also useful for examining mitochondrial activity, as well as protein expression in cells and tissues. Our work represents a progression in mitochondria research, and may be especially useful to researchers who have struggled with the isolation of mitochondria. Our methodological improvements may contribute to advanced research in the field by enabling investigators to overcome the difficulties of isolating mitochondria from biological samples.

\section{Acknowledgements}

This work was supported by the BK21 plus program through the National Research Foundation (NRF) funded by the Ministry of Education, and by the Bio-Meditech 
Regional Innovation Center at Eulji University, under the Regional Innovation Center Program (2013-02-10) of Ministry of Trade, Industry and Energy, Republic of Korea.

\section{REFERENCES}

Bratic A, Larsson N. The role of mitochondria in aging. J Clin Invest. 2014. 123: 951-957.

Frezza C, Cipolat S, Scorrano L. Organelle isolation: functional mitochondria from mouse liver, muscle and cultured fibroblasts. Nat Protoc. 2007. 2: 287-295.

Runkel ED, Baumeister R, Schulze E. Mitochondrial stress:
Balancing friend and foe. Exp Gerontol. 2014. 56: 194-201. Schwarz K, Siddiqi N, Singh S, Neil CJ, Dawson DK, Frenneaux MP. The breathing heart - Mitochondrial respiratory chain dysfunction in cardiac disease. Int J Cardiol. 2014. 171: 134 $-143$.

Sung HJ, Wenzhe M, Wang P, Hynes J, O'Riordan TC, Combs CA, McCoy JP, Bunz F, Kang J, Hwang PM. Mitochondrial respiration protects against from oxygen-associated DNA damage. Nat Commun. 2010. 1: 5.

Waris G, Ahsan H. Reactive oxygen species: role in the development of cancer and various chronic conditions. J Carcinog. 2006. 5: 14. 